Urologe 2012 $\cdot 51: 1063-1064$

DOI 10.1007/s00120-012-2933-3

Online publiziert: 22 . Juli 2012

○) Springer-Verlag 2012

\author{
A. Schroeder ${ }^{1}$ - S.C. Müller ${ }^{2}$ \\ 1 Urologische Praxis, Neumünster \\ ${ }^{2}$ Klinik und Poliklinik für Urologie, Universitätsklinikum Bonn, Bonn
}

\title{
Berufsbild und Sektorengrenzen im Wandel
}

zubauen, wird die eigentliche Herausforderung.

Die rechtlichen Voraussetzungen für die Überwindung dieser Grenzen sind erstmals in Vertragsarztänderungsgesetz, Bundesmantelvertrag und Versorgungsstrukturgesetz geschaffen worden, so etwa in den Bestimmungen zum Qualitätswettbewerb sowie in der ambulanten spezialfachärztlichen Versorgung ( $\$ 116 \mathrm{~b})$, der vor- und nachstationären Behandlung durch Vertragsärzte ( $\$ 115 \mathrm{a})$ und dem ambulanten Operieren ( $\$ 115 b)$.

Um Unter-, Fehl- und Überversorgung abzubauen und zu vermeiden, werden innovative Versorgungsformen benötigt. Aber auch die integrierte Versorgung muss wieder das Vertragsgerüst ergänzen. Grundsätzlich müssen Steuerungsinstrumente und Versorgungsstrukturen so justiert werden, dass die zentralen Prinzipien unseres Gesundheitssystems nicht in Frage gestellt werden.

Die Urologie muss hinzugewonnene Arbeits- und Therapiebereiche kompetent ausbauen. Dies betrifft v. a. medikamentöse Tumortherapie, Andrologie, gynäkologische Urologie, Kinderurologie, Männergesundheit sowie primäre Prävention. Von allen Krebspatienten in Deutschland werden bereits heute rund 25\% von Urologen behandelt, meist durch Operation und medikamentöse Tumortherapie, aber auch durch aktive Überwachung. Angesichts der Individualisierung in der Krebstherapie sollten diese multimodalen Konzepte zum Wohle des Patienten in einer Hand bleiben.
Die Überalterung der Gesellschaft wird den Bedarf an Behandlung und Pflege in der nahen Zukunft rapide zunehmen lassen. Schon jetzt leben in Deutschland etwa 700.000 pflegebedürftige Menschen in Heimen, Tendenz steigend. Rund $70 \%$ dieser Pflegebedürftigen sind harninkontinent und daher chronische Urologiepatienten. Sie benötigen im Rahmen der Pflegereform ein verbessertes Versorgungskonzept, das jedoch nur durch neuartige Kollektiv- und Selektivverträge in der Praxis der Heimversorgung erreichbar ist.

Angesichts des fortschreitenden demografischen Wandels kann davon ausgegangen werden, dass bei steigendem Bedarf an Leistungen in Gesundheit und Pflege und gleichzeitig knapper werdenden Mitteln der Zeitpunkt kommt, an dem Stichworte wie Priorisierung und Rationierung in den Vordergrund rücken. Hier sollten wir Ärzte uns von der Politik nicht den Schwarzen Peter für Leistungseinschränkungen zuschieben lassen. Nur nach ausschließlich medizinisch begründeter Indikationsstellung und auf Basis eines eindeutigen gesetzlichen Auftrags, ähnlich dem Prinzip beim Transplantationsgesetz, kann ein Weg zur medizinischen Priorisierung gangbar sein.

Schon jetzt steht dem steigenden Bedarf an Leistungen in der Gesundheitsversorgung in vielen ländlichen Regionen ein Ärztemangel in Praxis und Klinik gegenüber. Diese Entwicklung wird verschärft durch den „Frust des Alltags“ für Ärzte - zu viel Bürokratie und zu ge- in den Köpfen der Ärzte, in ihren Institutionen und bei den Krankenkassen ab- 
ring gewordene Verdienstmöglichkeiten locken nicht in den Beruf. Außerdem müssen sich fachlich ethische Kriterien der Berufsausübung immer öfter kommerziellem Druck von Geschäftsführern und Investoren beugen. Der Status des freien Arztberufes wird zunehmend zu einem Angestelltenverhältnis in einer "Gesundheitswirtschaft" abqualifiziert. Daran wird auch die Novellierung der Gebührenordnung für Ärzte (GOÄ) wenig ändern, so sie denn irgendwann kommt. Struktur und Leistungsbeschreibung der GOÄ tragen veraltete Züge, aber trotz dieses unzumutbaren und innovationsfeindlichen Zustands liefern deutsche Ärzte weiter Spitzenmedizin ab. Für die Verantwortlichen scheint somit kein Grund für Eile mit Veränderungen zu bestehen.

Der bereits erwähnte Ärztemangel betrifft besonders operative Fächer wie die Urologie. Zwar ist ein Medizinstudium weiter stark gefragt, es mangelt jedoch an objektiven Messmethoden für tatsächliche ärztliche Kompetenzen am Ende des Studiums. Auch bei der Weiterbildung zum Facharzt fehlen gerade in operativen Fächern standardisierte und nachprüfbare Konzepte. In Deutschland werden Versuche anderer Länder, operative Fähigkeiten und manuelle Geschicklichkeit objektiv zu erkennen und zu fördern, bisher ignoriert. Hier besteht Nachholbedarf, um den Anschluss nicht zu verpassen.

Vielfältig sind dagegen die konzeptionellen Ansätze und Überlegungen zur Verbesserung der Patientenversorgung: Die Sinnhaftigkeit leitlinienorientierter Medizin steht außer Frage. In diesem Heft wird beschrieben, wie durch konsequente Umsetzung von Leitlinien eine Reduktion der Rezidiv-, Morbiditäts- oder Mortalitätsrate mit vertretbarem Aufwand erreichbar ist. Leitlinien, die keiner Normierung unterliegen und mit immensen Kosten erstellt werden, spiegeln häufig nur den kleinsten gemeinsamen Nenner wider, und teilweise gibt es auch erhebliche Unterschiede zu denen anderer Länder. Daher wären internationale Leitlinien sinnvoll, um z. T. sich widersprechende Konzepte zu vermeiden. Zudem wäre ihre geforderte Aktualisierung im 2-jährigen Turnus durch die Verteilung auf mehr Schultern mit weit geringerem Aufwand möglich.

Ein weiterer Weg zur Optimierung der Behandlung wird mit der Untersuchung der Interdisziplinarität begangen: So wird von Patientenselbsthilfegruppen, aber auch in den Onkologievereinbarungen von jeher eine verbesserte Zusammenarbeit von Urologen und Onkologen gefordert. Wie es bundesweit um die tägliche Praxis dieser Kooperation steht, weiß bisher niemand so genau. Hier soll die als multizentrische Erhebung angelegte IBuTu-Studie (interdisziplinäre Behandlung urologischer Tumoren) Abhilfe schaffen.

Einen ganz anderen Ansatz zur besseren Patientenversorgung, besonders ihrer Sicherheit, stellt CIRS („critical incident reporting system“) dar. Dieses Fehlermanagementsystem bietet die Möglichkeit, eigene Fehler anonym zu veröffentlichen, um dadurch eine Wiederholung derselben Fehler durch Kollegen zu vermeiden und die Sicherheit ihrer Patienten zu erhöhen. Das niedrigschwellige Online-System hat sich bereits in anderen Bereichen bewährt und wird im Herbst vom Berufsverband der Deutschen Urologen implementiert.

Insgesamt muss sich die Urologie keine massiven Zukunftssorgen machen, wenn wir den Autoren dieses Heftes folgen. Die Urologie muss sich nicht prinzipiell neu auf den demographischen Wandel einstellen, denn sie ist ein generell geriatrisches und ein in weiten Teilen onkologisches Fachgebiet. Zudem bietet sie als integratives Fachgebiet Versorgung aus einer Hand und ist zur interdisziplinären Kooperation bereit. Für notwendige Anpassungsprozesse ist die Urologie gerüstet und will sie aktiv mitgestalten, um sich bietende Chancen und Perspektiven für die Versorgung zu nutzen.

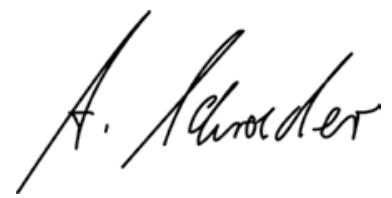

A. Schroeder (BDU-Präsident)

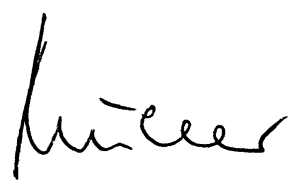

S.C. Müller (DGU-Präsident)

\section{Korrespondenzadressen}

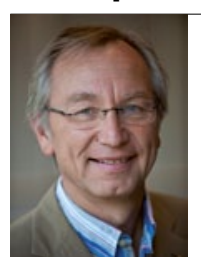

Dr. A. Schroeder

Urologische Praxis,

Haart 87-89,

24534 Neumünster

drschroeder@

bdu-praesident.de

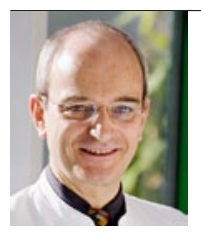

Prof. Dr. Dr. h.c. S.C. Müller

Klinik und Poliklinik

für Urologie,

Universitätsklinikum Bonn,

Sigmund-Freud-Straße 25,

53105 Bonn 